\title{
EFFECT OF MENISECTOMY ON RECOVERY OF PROPRIOCEPTIVE DEFICITS FOLLOWING ARTHROSCOPIC ACL RECONSTRUCTION
}

\author{
Atul Mahajan *1, Anil Mehtani ${ }^{2}$. \\ ${ }^{*}$ Assistant Professor, Department of Orthopedics, Lady Hardinge Medical College, New Delhi, \\ India. \\ 2 Professor, Lady Hardinge Medical College, Delhi, India.
}

\section{ABSTRACT}

\begin{abstract}
Introduction: Decrease in proprioception and kinesthesia occurs after ACL injury. Changes occurring within the joint following injury affect normal recruitment and firing patterns of the surrounding musculature. There are little data in the literature with reference to effect of concomitant menisectomy on recovery of proprioceptive deficits after arthroscopic $\mathrm{ACL}$ reconstruction.

Objective: To determine the effect of concomitant menisectomy on proprioception recovery after arthroscopically assisted anterior cruciate ligament $(\mathrm{ACL})$ reconstruction with quadrupled hamstring graft.

Materials and Methods: In this study, 42 arthroscopic ACL reconstruction patients were randomized into Group $A C L+M M$ which underwent $A C L$ reconstruction and menisectomy for concomitant meniscal tears and Group $A C L$ who underwent $A C L$ reconstruction alone. Outcome Assessment was done at 1 month, 3, 6 and 12 months after the surgery in which patients were assessed using Star excursion balance test by measuring excursion distances in each of the 8 directions.

Results: Excursion distances in eight directions showed less increment inACL+MM group with compared to $A C L$ group. However it was found to be statistically significant only for posteromedial, lateral and medial directions.

Conclusion: Concomitant menisectomy does affect the recovery of dynamic proprioceptive deficits and the recovery phase is longer after arthroscopically assisted anterior cruciate ligament $(\mathrm{ACL})$ reconstruction compared to $A C L$ reconstruction alone.

KEY WORDS: Star excursion balance test, Anterior cruciate ligament, Dynamic proprioception, Postural Stability.
\end{abstract}

Address for correspondence: Dr. Atul Mahajan, Assistant Professor. Department of Orthopedics, Lady Hardinge Medical College, New Delhi, India. Ph 919643597479

E-Mail: dratulmahajan84@gmail.com

\begin{tabular}{|c|c|c|}
\hline \multicolumn{3}{|c|}{ Access this Article online } \\
\hline 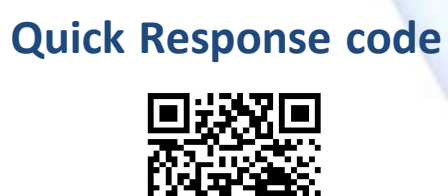 & \multicolumn{2}{|c|}{$\begin{array}{l}\text { International Journal of Physiotherapy and Research } \\
\text { ISSN 2321- } 1822 \\
\text { www.ijmhr.org/ijpr.html }\end{array}$} \\
\hline DOI: $10.16965 /$ ijpr.2017.178 & $\begin{array}{l}\text { Received: 24-05-2017 } \\
\text { Peer Review: 25-05-2017 } \\
\text { Revised: None }\end{array}$ & $\begin{array}{l}\text { Accepted: } 19-06-2017 \\
\text { Published (O): 11-08-2017 } \\
\text { Published (P): 11-08-2017 }\end{array}$ \\
\hline
\end{tabular}

\section{INTRODUCTION}

The Anterior cruciate ligament plays an important role in the stability of the knee primarily through its passive constraint to anterior tibial translation and tibial rotation. Also it influences the dynamic function of the knee [1]. Knee injuries and knee surgery cause damage to part of the mechanoreceptors needed to collect proprioceptive information. Studies suggest that impaired knee proprioception may increase the risk of micro-trauma, thereby leading to early degenerative knee lesions [2,3].

Decrease in proprioception and kinesthesia occurs after ACL injury. Changes that occur within the joint affect normal recruitment and timing patterns of the surrounding musculature. In the ACL deficient (ACLD) knee, movement occurs in a non-physiological axis, creating alterations in 
gait and movement [4]. The sensation of instability and giving way that the ACLD patient describes has been attributed to poor proprioception [5] in addition to the actual functional instability. ACL injury has been associated with a resultant decrease in proprioceptive performance, with this relationship between $A C L$ rupture and decreased proprioception being reported to be due to damage to the mechanoreceptors in the articular structures and ACL [6].

Studies have also indicated $[7,8]$ that knee proprioception is decreased in both partially and totally meniscectomized knees due to the loss of mechanoreceptors present in the resected meniscus.

However, it is unclear whether postural stability would be further reduced in patients who also have meniscal tears combined with $A C L$ tears compared to $A C L$ tears alone and extent of recovery and progression following surgical intervention. So, we conducted this study to determine the effect of concomitant menisectomy on proprioception recovery after arthroscopically assisted anterior cruciate ligament $(\mathrm{ACL})$ reconstruction with quadrupled hamstring graft.

\section{MATERIALS AND METHODS}

This prospective longitudinal trial enrolled patients who were candidates for ACL reconstruction between 2015 and 2016 were included in the study. Patients were included only if they had $A C L$ ruptures associated with medial meniscus tears ( $A C L+M M$ group) or unilateral isolated $A C L$ ruptures (ACL group) based on the $\mathrm{MRI}$ and arthroscopic findings. They were then followed up for the duration of one year post operatively. Patients with $A C L$ tear presenting after six weeks but before six months from the date of injury, patients with $A C L$ tear who had minimum of 120 degrees of flexion possible at the knee before surgery and had no pain in the affected knee were included in the study. Patients with multi ligament injuries, Presence of fractures or deformities in the lower limb, revision anterior cruciate ligament reconstruction were excluded. Patients associated with lateral meniscus tears were excluded to eliminate bias resulting from meniscus location. Individuals with prior experience of dynamic balance testing were excluded to eliminate a learning curve effect.

Proprioception analysis was assessed using star excursion balance test (SEBT). Patients who were to take the test were selected based if they had no history of ankle injury, no history of cerebral concussions, no ear infection or upper respiratory infection at the time of test, no prior balance training. To perform the SEBT, subjects were asked to stand on one foot and reach as far as they could in each of 8 different directions with the other foot, touching down lightly on the line, then return to the starting position as shown in (Figure 1). Subjects maintained a single-limb stance until they returned to the starting position. The 8 directions extend out in a circle and each target line is $45^{\circ}$ from the adjacent lines. Each direction is completed as a separate trial and reach distance is recorded in centimeters. Three practice trials were permitted for each lower extremity to minimize learning effect. Subjects were then allowed to rest and completed 3 measured trials on each lower extremity. The mean reach distance for each lower extremity was recorded. Trials were discarded and repeated if the hands were removed from the hips, the reaching limb was used for weight bearing, the stance limb was displaced or there was a loss of balance.

Fig. 1: The 8 positions of the SEBT are based on the stance of the affected limb.
Left Limb Stance

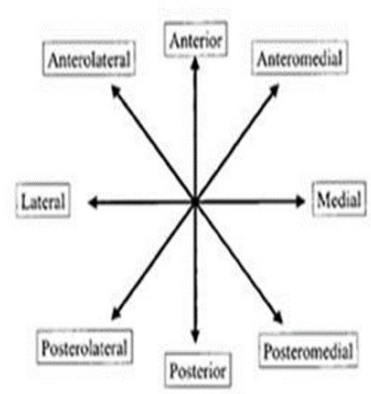

Right Limb Stance

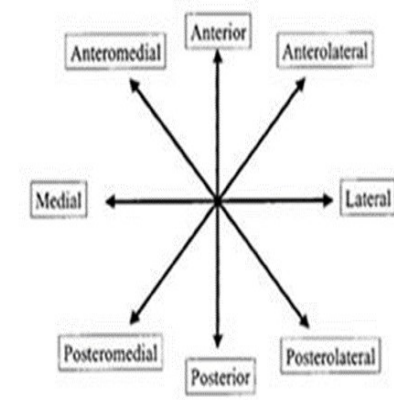

The type of anesthetic (regional or general) was not controlled and was selected at the discretion of the anesthesiologist. All patients received prophylactic intravenous antibiotics preoperatively. A pneumatic tourniquet was used following exsanguination to produce a relatively bloodless operative field. Same team of surgeons performed all of the ligament reconstructions 
in the patients in this study. The anterior cruciate ligament was reconstructed with quadrupled hamstring graft, harvested from the ipsilateral knee under tourniquet. Trans-tibial technique was used in graft placement. Anchorage of the graft was done using bio-absorbable interference screws at tibial side and either cross pins or endobutton for femoral side. The knee was closed primarily and 3 layered compressive dressing was placed on the knee of all patients. A knee immobilizer was applied for all the patients for first 2 weeks at rest. Patients were allowed partial weight bearing from the next day after gait training with bilateral axillary crutches. All patients were encouraged to elevate the extremity when not performing therapy. Postoperative rehabilitation was performed in accordance with the accelerated $\mathrm{ACL}$ protocol and was identical in both the groups. Similar postoperative analgesia regimen was followed. Assessment was done on postoperative 1, 3, 6 and 12 months after the surgery based on the same criteria considered preoperatively. Results were expressed as mean \pm SD. Statistical analysis was performed by using repeated-measures analysis of variance (ANOVA). Independent sample t-test was used to compare the treatment group and control group over a period of time. The difference between the two groups was considered significant if the $p$ value was $<0.05$.

\section{RESULTS}

There were 46 patients who met the inclusion criteria for the study. Four were lost to followup after surgery. Therefore, 42 patients were alternatively allocated either the $\mathrm{ACL}+\mathrm{MM}$ group (21) or the ACL group (21).

The final population (Table 1) comprised 36 men $(A C L+M M$ group=19; $A C L$ group=17) and 6 women ( $A C L+M M$ group=2; $A C L$ group $=4)$ with a mean age of $32.85+/-5.68$ years ( $A C L+M M$ group $=32.65 \pm 5.43 ; \mathrm{ACL}$ group=33.05 \pm 5.93$)$. Those less than 28 years of age were comprised mainly of students. The gender distribution of cases was eccentric with more males than females in the study. This can be explained on the basis of lower rates of road traffic accidents among females and lesser participation in sports activities. The mean body mass index was $25.71+/-3.26 \mathrm{~kg} / \mathrm{m} 2$ (ACL+MM group $=26.19 \pm$
3.75; $A C L$ group $=25.24 \pm 2.77)$. The mean duration of symptoms was 9 months $(\mathrm{ACL}+\mathrm{MM}=$ $8.65 \pm 2.44 ; \mathrm{ACL}=9.7 \pm 1.47$ ).

Table1: Demographic characters of the study group.

\begin{tabular}{|l|c|c|}
\hline \multicolumn{1}{|c|}{ Variables } & ACL+MM & ACL \\
\hline No. of patients & 21 & 21 \\
\hline Female: male & $2: 19$ & $4: 17$ \\
\hline Age & $32.65 \pm 5.43$ & $33.05 \pm 5.93$ \\
\hline BMI & $26.19 \pm 3.75$ & $25.24 \pm 2.77$ \\
\hline Right: Left & $8: 13$ & $11: 10$ \\
\hline Duration months & $8.65 \pm 2.44$ & $9.7 \pm 1.47$ \\
\hline
\end{tabular}

8 operations in the right and 13 in the left knee were performed in the $A C L+M M$ group and 11 in the right and 10 in the left knee in the $A C L$ group. No infection was observed after surgery in both groups. The study group had $71 \%(n=30)$ patients who were involved in sports and outdoor activities before injury compared to $20 \%$ $(n=12)$ patients who were involved in sedentary occupation.

Pre-operative mean excursion distances (Table 2) showed decline in $A C L+M M$ group when compared to the opposite limb and these differences were statistically significant in all the directions. $(p<0.05)$ In $A C L$ group also, mean excursion distances were less compared to normal opposite limb taken as control and differences were statistically significant $(p<0.05)$ except for anterior and lateral directions. (ANT:0.26; L: 0.08)

Table 2: Mean excursion distance comparison between affected limb and normal unaffected contralateral limb in $A C L+M M$ and $A C L$ groups in 8 different directions.

\begin{tabular}{|c|c|c|c|c|}
\hline ACL+MM & & AFFECTED & UNAFFECTED & P VALUE \\
\hline & ANT & 78.82 & 82.5 & 0.007 \\
\hline & AM & 74.14 & 79.18 & 0.001 \\
\hline & AL & 79.5 & 83 & $<0.001$ \\
\hline & L & 74.91 & 81.73 & 0.001 \\
\hline & POST & 74.18 & 83.59 & $<0.001$ \\
\hline & PM & 72.86 & 78.95 & 0.005 \\
\hline & PL & 77.18 & 81.95 & $<0.001$ \\
\hline ACL & M & 63 & 65.95 & $<0.001$ \\
\hline & & & & \\
\hline & ANT & 84.95 & 86.73 & 0.26 \\
\hline & AM & 76.77 & 74.14 & 0.126 \\
\hline & AL & 84.14 & 89.36 & 0.004 \\
\hline & L & 82.27 & 86.67 & 0.085 \\
\hline & POST & 71.64 & 77.77 & $<0.001$ \\
\hline & PM & 67.23 & 73.64 & 0.011 \\
\hline & PL & 78.95 & 83.45 & 0.002 \\
\hline & M & 52.45 & 64.23 & $<0.001$ \\
\hline
\end{tabular}

(ANT=Anterior; $A M=$ Anteromedial; $A L=$ Anterolateral; $\mathrm{L}=$ Lateral; $\mathrm{POST}=$ Posterior; $\mathrm{PM}=$ Posteromedial;

$\mathrm{PL}=$ Posterolateral; $\mathrm{M}=$ Medial) 
When followed up the patients (Table 3) over a period of 12 months, $A C L+M M$ group showed improvement in excursion distances in all the directions compared to pre- operative values. These difference was clinically significant in all directions and statistically significant in Medial, Lateral, Posterior, Posteromedial and posterolateral directions. $(p<0.05)$

Table 3: Mean excursion distance improvement over a period of follow-up of 12 months in $A C L+M M$ and $A C L$ groups in 8 different directions.

\begin{tabular}{|c|c|c|c|c|c|c|}
\hline ACL+MM & PRE OP & 1 MONTH & 3 MONTHS & 6 MONTHS & 12 MONTHS & P VALUE \\
\hline ANT & 78.82 & 78.77 & 79.45 & 76.05 & 79.82 & 0.435 \\
\hline AM & 74.14 & 74.82 & 75.82 & 75.27 & 75.18 & 0.711 \\
\hline AL & 79.5 & 78.86 & 78.86 & 79.36 & 79.27 & 0.933 \\
\hline L & 74.91 & 74.14 & 76.14 & 78.68 & 77.59 & 0.004 \\
\hline POST & 74.18 & 71.27 & 72.18 & 73.95 & 75.82 & 0.043 \\
\hline PM & 72.86 & 67.41 & 70.32 & 74.86 & 74.9 & $<0.001$ \\
\hline PL & 77.18 & 77.27 & 78.27 & 79.86 & 79.45 & 0.04 \\
\hline M & 63 & 54.09 & 57.23 & 61.68 & 62.64 & $<0.01$ \\
\hline ACL & & & & & & \\
\hline ANT & 84.95 & 81 & 81.86 & 84.32 & 84.77 & 0.002 \\
\hline AM & 76.77 & 75.68 & 76.32 & 77.45 & 79.36 & 0.002 \\
\hline AL & 84.14 & 81.27 & 82.27 & 83.05 & 86.18 & 0.001 \\
\hline L & 82.27 & 78.23 & 79.41 & 82 & 81.82 & 0.047 \\
\hline POST & 71.64 & 69.5 & 70.41 & 72.36 & 75.05 & $<0.001$ \\
\hline PM & 67.23 & 63.86 & 64.86 & 68.68 & 72.41 & $<0.001$ \\
\hline PL & 78.95 & 77.14 & 77.77 & 79.64 & 80.36 & 0.06 \\
\hline M & 52.45 & 49.41 & 53.68 & 57.18 & 63.41 & $<0.01$ \\
\hline ACL
\end{tabular}

ACL group also showed improvement in excursion distances in all the directions which was clinically significant in all directions and statistically significant $(p<0.05)$ in all except posterolateral directions. $(p=0.06)$

On comparing final mean excursion distances (Table 4) of $\mathrm{ACL}+\mathrm{MM}$ group with $\mathrm{ACL}$ group, all the directions in $A C L$ group showed more clinical increment compared to ACL+MM group. However, this clinical improvement was statistically significant in three of the eight directions: posteromedial; medial and lateral direction. ( $p$ for $\mathrm{PM}=0.018$; $\mathrm{M}<0.01 ; \mathrm{L}=0.034$ )

Table 4: Comparison between $\mathrm{ACL}+\mathrm{MM}$ group and $\mathrm{ACL}$ group after 12 months.

\begin{tabular}{|c|c|}
\hline Direction & p-value \\
\hline AM & 0.159 \\
\hline ANT & 0.52 \\
\hline AL & 0.102 \\
\hline L & 0.034 \\
\hline PL & 0.508 \\
\hline POST & 0.22 \\
\hline PM & 0.018 \\
\hline M & $<0.001$ \\
\hline
\end{tabular}

\section{DISCUSSION}

One of the most frequently damaged structures in the knee is the anterior cruciate ligament $(A C L)$, particularly in young people who are physically active. Ligament injuries are usually accompanied with damage to one or both menisci and/or to the articular cartilage. Injuries are 5 times more common in the medial meniscus (MM) than in the lateral meniscus. In most cases, injuries of the $A C L$ and $M M$ result in impaired knee proprioception [9].

The concentration of the mechanoreceptors involved in proprioception is highest in the posterior corner of the $\mathrm{MM}$ and at the proximal and distal attachments of the cruciate ligaments to the bones $[10,11]$.

Injuries to ligaments, one or both menisci, and/ or the articular cartilage in the knee cause partial damage to the mechanoreceptors contained in these structures, thereby inducing disorders in both static proprioception and kinaesthesia $[12,13]$. Another consequence is mechanical instability, which can further impair body balance [2]. Therefore, ACL and MM injuries usually require both surgical treatment and specialised rehabilitation emphasising proprioception training [14].

Neuromuscular training is typically used to enhance athlete's preparation, performance and recovery by improving dynamic postural stability which is an integral component of lower limb neuromuscular control. Whereas static measures of postural-control provide useful clinical information, the underlying task of standing as still as possible might not translate necessarily to movement tasks during physical activity. Conversely, dynamic postural-control involves some level of expected movement around a base of support.

Most studies of the impact of meniscus tears on knee joint proprioception have used joint position sense tests, with joint position measured after partial or total meniscectomy. A case-control study comparing 19 patients with medial meniscus posterior horn tears at a mean 2 years after partial meniscectomy with 20 healthy individuals on knee angle reproduction tests found that the former group showed more deviation than did the control group, indicating 
poorer proprioception in the meniscectomized than in the control group [15].

Evaluation of the change in joint position sense in patients who underwent meniscus allograft transplantation (MAT) for totally meniscectomized knees showed that, compared with preoperative measurements, proprioception significantly improved 6 months after MAT [16]. Taken together, these studies indicate that knee proprioception is decreased in both partially and totally meniscectomized knees due to the loss of mechanoreceptors present in the resected meniscus.

Few studies have evaluated the effect of meniscus tears on postural stability. A comparison of postural stability using the balance index in subjects with and without meniscus tears found that overall balance performance was significantly better in participants without meniscus tears [17]. In contrast, a prospective posturographic evaluation of postural stability in 27 patients with unilateral meniscal lesions showed no differences in stability indexes on the injured and uninjured sides [18].

These discrepancies in different studies may be due to differences in the location and extent of meniscus tears, differences in subject age or sports activities, and to the lack of a standardized measurement system or calculation method. In our study, we used Star Excursion Balance test( SEBT) which is a promising test of dynamic postural control that may be useful in assessing functional deficits. Completion of the SEBT requires many attributes including strength, flexibility, neuromuscular control, core stability, range of motion, balance and proprioception [19]. Utilizing this test can provide us with objective data for dynamic postural stability evaluation for use in the areas of injury prevention and management, namely assessment, rehabilitation, screening. Each reaching direction offers different challenges and requires combinations of sagittal, frontal, and transverse movements. Earl and Hertel [20] found that muscle activation was substantially different across the various reach directions, activity being higher during the posterior, posterolateral, and lateral excursions than during the anterior and anteromedial excursions. This finding might be helpful to clinicians deciding which reach directions to employ as outcome measures in patients with specific impairments in muscle strength. In our study, it was found that significant differences were seen in medial, lateral, posteromedial directions indicating these directions can be used as an outcome measure to diagnose impairments in ACL with or without meniscal tears. The most important finding of the present study was that postural stability on the affected sides did differ significantly between patients with isolated $A C L$ tears and those with both $A C L$ tears and meniscal tears. This difference was significant clinically in all directions and statistically significant in medial, lateral and posteromedial directions.

Postural stability is decreased to some extent in patients with ACL tears [21], presumably due to disruption of mechanoreceptors within the ligament. That is, a slight decrease in postural stability resulting from a meniscus tear may be masked by the greater reduction resulting from an ACL tear. Thus, postural instability on the affected side may be similar in these two groups of patients.

In our study, postural control was more impaired and slow to recover among patients who had had $A C L$ reconstruction combined with menisectomy compared to patients with isolated ACL reconstructions.

Reduced balancing ability on the affected side was most likely to be caused by deficits due to mechanoreceptor injuries [22]. In the postoperative period, balancing ability increased continuously in both the groups over a period of follow up. This increase was more in isolated $A C L$ group compared to $A C L+M M$ group indicating that patients with combined $A C L$ and medial meniscus tears were less capable of adapting to unidirectional perturbation and had slower recovery in postural stability. Also, the removal of damaged mechanoreceptors in partial medial meniscectomy may reduce balancing capacity. However, Improvement in postural stability did not reach the level of normal unaffected limb, even one year after surgery. This might reflect interference between damaged and healthy mechanoreceptors, which may reduce the overall balancing capacity. From our results it can be inferred that the one-year postoperative period was not enough for the regeneration of 
mechanoreceptors in the meniscus and thus special emphasis should be placed on improving both static and dynamic balance when arranging postoperative rehabilitation.

This study had several limitations, one of the most important being the differences in meniscus tear patterns and extent among the subjects studied which was not taken into account. Although the meniscus has receptors in all Cooper zones, the red and red-white zones contain more receptors than the white zone [15]. Therefore, tears involving the peripheral areas may result in injury to more mechanoreceptors, such that postural stability would be poorer in patients with peripheral than inner rim tears.

In addition, only patients with medial meniscus tears were included in the present work to avoid any potential difference in postural stability between patients with medial and lateral meniscus tears. This may have resulted in some selection bias. However, the dominance in this study of patients with chronic ACL tears led to the exclusion of patients with lateral meniscal tears, because medial meniscal tears are more common in patients with chronic than acute $A C L$ tears [23], whereas lateral meniscus tears are more common in patients with acute $A C L$ tears [24]. The last limitation of this research is that electromyographical analysis was not used to determine the response of the muscles surrounding the knee. In further research muscle response could be analyzed.

\section{CONCLUSION}

Concomitant menisectomy does affect the recovery of dynamic proprioceptive deficits and the recovery phase is longer after arthroscopically assisted anterior cruciate ligament $(A C L)$ reconstruction compared to $A C L$ reconstruction alone.

\section{Conflicts of interest: None}

\section{REFERENCES}

[1]. Noyes, F. R.; Grood, E. S.;Butler, D. L. Clinical laxity tests and functional stability of the knee: biomechanical concepts. ClinOrthop 1980;146:84-89.

[2]. Henriksson M, Ledin T, Good L. Postural control after anterior cruciate ligamentreconstruction and functional rehabilitation. Am J Sports Med 2001;29:359- 66.
[3]. Riemann BL, Lephart SM. The sensorimotor system, part I: the physiologic basisof functional joint stability. J Athl Train 2002;37:71-9.

[4]. Edwards, A.; Bull, A.; Amis , A. The attachments of the anteromedial and posterolateral fibre bundles of the anterior cruciate ligament: part 2: femoral attachment. Knee Surg Sports Traumatol Arthros 2008;16:29-36.

[5]. Peterson,W.;Zantop,T. Anatomy of anterior cruciate ligament with regard to its two bundles. Clin Orthop Relat Res 2007;454:35-47.

[6]. Limbird TJ, Shiavi R, Frazer M, Borra. HEMG profiles of knee joint musculature during walking: changes induced by anterior cruciate ligament deficiency. J Orthop Res. 1988; 6(5):630-8.

[7]. Brindle T, Nyland J, Johnson DL. The meniscus: review of basic principles with application to surgery and rehabilitation. J Athl Train 2001;36:160169.

[8]. Aagaard H, Verdonk R. Function of the normal meniscus and consequences of meniscal resection. Scand J Med Sci Sports 1999;9:134-140.

[9]. Ahmed MM, Mosalem DM, Khairet SM, Ismail TA, Hamido FA, Al-Busairi WA. Evaluation of dynamic posturography in anterior cruciate ligament injurypatients. Maced J Med Sci 2011;15:167-73.

[10]. Cho SR, Kim HM, Lee KH, Kim YS, Lee JH, Jahng YJ. The change of the mechanore-ceptor of anterior cruciate ligament in fixed knee and injured anterior cruciateligament in the rabbit. J Korean Orthop 2004;39:572-8.

[11]. Kanemura N, Kobayashi R, Kajihara H, Minematu A, Sasaki H, Tanaka S, et al. Changes of mechanoreceptor in anterior cruciate ligament with hindlimb sus-pension rats. J Phys Ther Sci 2002;14:27-32.

[12]. Rymer T, Kruczy'nski J. Knee joint proprioception evaluation with own con-struction device. Chir Narzadow Ruchu Ortop Pol 2007;72:189-92.

[13]. Dhillon MS, Bali K, Prabhakar S. Proprioception in anterior cruciate ligamentdeficient knees and its relevance in anterior cruciate ligament reconstruction.Indian J Orthop 2011;45:294-300.

[14]. Pasierbi'nski A, Jarz²abek A. Rehabilitation after anterior cruciate ligamentreconstruction. Acta Clinica 2002;2:86-100.

[15]. Karahan M, Kocaoglu B, Cabukoglu C, Akgun U, Nuran R. Effect of partial medial meniscectomy on the proprioceptive function of the knee. Arch Orthop Trauma Surg 2010;130:427-431.

[16]. Thijs $Y$, Witvrouw E, Evens B, Coorevits P, Almqvist F, Verdonk R. A prospective study on knee proprioception after meniscal allograft transplantation. Scand J Med Sci Sports 2007;17:223-229.

[17]. Lange AK, Singh F, Smith RM, Foroughi N, Baker MK, Shnier R, Vanwanseele B. Degenerative meniscus tears and mobility impairment in women with knee osteoarthritis. Osteoarthr Cartil 2007;15:701-708.

[18]. Palm HG, Laufer C, von Lu“bken F, Achatz G, Friemert B. Do meniscus injuries affect postural stability? Der Orthopade 2010;39:486-494. 
[19]. Gribble, PA., Hertel, J., and Plisky, P. Using the Star Excursion Balance Test to assess dynamic posturalcontrol deficits and outcomes in lower extremity injury: a literature and systematic review. Journal of Athletic Training, 2012;47:339-357.

[20]. Earl, J., and Hertel, J. Lower-extremity muscle activation during the Star Excursion Balance Tests. Journal of Sports Rehabilitation 2001;10:93-104.

[21]. Kennedy JC, Alexander IJ, Hayes KC. Nerve supply of the human knee and its functional importance. Am J Sports Med 1982;10:329-335.

[22]. Al-Dadah O, Shepstone L, Donell ST. Proprioception following partial meniscectomy in stable knees. Knee Surg Sports Traumatol Arthrosc 2011;19:207213.
[23]. Wang JH, Wong AK, Romanowski JR 2nd, Fu FH. Meniscus tear developed by pulling of the anomalous insertion of medial meniscus on anterior cruciate ligament. Knee Surg Sports Traumatol Arthrosc 2011;19:1689-1692.

[24]. Shelbourne KD, Nitz PA. The O'Donoghue triad revisited. Combined knee injuries involving anterior cruciate and medial collateral ligament tears. Am J Sports Med 1991;19:474-477.

How to cite this article:

Atul Mahajan, Anil Mehtani. EFFECT OF MENISECTOMY ON RECOVERY OF PROPRIOCEPTIVE DEFICITS FOLLOWING ARTHROSCOPIC ACL RECONSTRUCTION. Int J Physiother Res 2017;5(4):2225-2231. DOI: 10.16965/ijpr.2017.178 